\section{Autoavaliação da saúde bucal e fatores associados entre adultos em áreas de assentamento rural, Estado de Pernambuco, Brasil}

\author{
Self-rated oral health and associated factors among \\ adults in rural settlements, Pernambuco State, \\ Brazil
}

\author{
Autoevaluación de la salud bucal y factores \\ asociados entre adultos en áreas de asentamiento \\ rural, estado de Pernambuco, Brasil
}

\begin{abstract}
The aim of this study was to estimate prevalence of negative self-rated oral health and associated factors among adults in rural settlements. The probabilistic sample consisted of 557 adults 20 to 59 years of age in rural settlements in Pernambuco State, Brazil. The dependent variable was self-rated oral health, with the following independent variables: demographic characteristics, predisposition and availability of resources, oral health-related behavior, objective oral health conditions, and subjective oral health conditions. Crude and adjusted prevalence ratios were estimated using a Poisson regression model. Prevalence of negative self-rated oral health was 70.5\%. Negative self-rated oral health was associated with younger age, lower schooling, female gender, and black or brown skin color. Predictors of negative self-rated oral health included skin color, self-defined need for dental care, and the impact of oral health problems on quality of life.
\end{abstract}

Self-assessment; Rural Population; Oral Health; Cross-sectional Studies; Adult

\author{
Cristiano Moura ${ }^{1}$ \\ Estela Santos Gusmão 2 \\ Patrícia Morgana Hordonho Santillo 3 \\ Renata de Souza Coelho Soares 4 \\ Renata Cimões ${ }^{5}$
}

\section{Resumo}

O objetivo deste estudo foi estimar a prevalência de autoavaliação negativa de saúde bucal e fatores associados entre adultos em áreas de assentamento rural. A amostra probabilística consistiu de 557 adultos entre 20 a 59 anos em áreas de assentamento rural no Estado de Pernambuco, Brasil. A variável dependente foi autoavaliação da condição de saúde bucal e as independentes foram: características demográficas, de predisposição/disponibilidade de recursos, comportamentos relacionados à saúde bucal, condições objetivas e subjetivas relacionadas à saúde bucal. Foram estimadas as razões de prevalência bruta e ajustada por meio de regressão de Poisson. A prevalência de autopercepção negativa da saúde bucal foi de 70,5\%. A autoavaliação negativa da saúde bucal foi mais prevalente em indivíduos mais jovens, de baixa escolaridade, entre as mulheres, e entre os de cor preta e parda. Os preditores da autoavaliação negativa da saúde bucal foram a cor da pele, a necessidade autorreferida de tratamento odontológico e o impacto das condições de saúde bucal na qualidade de vida.

Autoavaliação; População Rural; Saúde Bucal; Estudos Transversais; Adulto 


\section{Introdução}

A autoavaliação da saúde bucal é uma medida multidimensional que reflete a experiência subjetiva dos indivíduos e sintetiza a condição objetiva da saúde bucal em termos de funcionalidade, valores sociais e culturais relacionados à essa 1 . Desta forma, entender o conceito subjetivo da saúde bucal possibilita investigar quais são os fatores associados à autoavaliação positiva ou negativa da condição de saúde bucal na população em estudo.

A autoavaliação negativa da saúde em geral, e nesta está inclusa a saúde bucal, pode ser vista como resultado de sentimentos provocados pela dor ou desconforto, pelo mal-estar, em interação com fatores sociais, culturais, psicológicos e ambientais que modificam a maneira como a vida da pessoa é afetada pelo problema experimentado ${ }^{2}$. Sendo assim, essa autoavaliação deve ser analisada sob uma ótica de múltiplos fatores, levando em consideração os diferentes entendimentos individuais de saúde, diante do contexto cultural, psicossocial e ambiental existente 3 .

Neste sentido, Gift et al. 4 elaboraram um modelo explicativo para a autoavaliação da saúde bucal, adaptado por Martins et al. 5, que considera a interação de cinco subgrupos de variáveis: características demográficas; elementos de predisposição/disponibilidade de recursos; comportamentos relacionados à saúde bucal; e condições objetivas e subjetivas relacionadas à saúde bucal, na elucidação desta autoavaliação. Neste modelo, existe uma relação de retroalimentação entre os cinco grupos de variáveis supracitados. Desta forma, as características demográficas, de predisposição e de disponibilidade de recursos, influenciam os comportamentos relacionados à saúde, que por sua vez podem predizer ou mesmo ser consequências das condições objetivas e subjetivas relacionadas à saúde geral e bucal. As condições objetivas influenciam as condições subjetivas e vice-versa, essas que, por sua vez, influenciam na autoavaliação da saúde bucal e geral.

No Brasil, diversos estudos se propuseram a investigar as desigualdades sociais em saúde bucal por meio da autoavaliação desta, entre adultos 6,7 e idosos 5,8,9, estritamente em áreas urbanas. Alguns desses estudos 4,7 têm demonstrado que a autoavaliação da saúde bucal varia de acordo com características sociodemográficas. Da mesma forma, pesquisas têm evidenciado associações da autoavaliação da saúde bucal com a utilização de serviços odontológicos 10; com as condições normativas de saúde bucal, dentre elas, o número de dentes perdidos, o número de dentes cariados e obturados, bem como o uso e necessidade de prótese 3,4; com a autopercepção da necessidade de tratamento odontológico 4. No entanto, é válido ressaltar que em se tratando de áreas rurais, poucos estudos nacionais $11,12,13$ buscaram retratar a percepção subjetiva em relação à saúde bucal.

Desta forma, muito pouco se conhece quanto às áreas de assentamento rural, definidas pelo Instituto Nacional de Colonização e Reforma Agrária (INCRA. http://www.incra.gov.br) como unidades empresariais associativas, de base familiar, autônomas e geridas pelos trabalhadores, que visam ao desenvolvimento econômico e social do conjunto de assentados. De fato, nos locais onde os assentamentos se consolidaram, rendas superiores são geradas em relação àquelas em atividades equivalentes, sob outras formas de exploração da mesma área 14. No entanto, esta situação não é uniforme para todos os assentamentos do país, nos quais se observa uma grande variabilidade nas condições socioeconômicas entre essas áreas e entre as famílias em um mesmo assentamento, a depender da região onde se localiza. É válido ressaltar a importância da organização do Movimento dos Trabalhadores Rurais Sem Terra (MST) no processo de reforma agrária no Brasil, que surge como uma resposta às profundas transformações econômicas $\mathrm{e}$ formas de expropriação no campo, decorrentes da modernização agrícola dos anos do milagre econômico, situação que se agravou com a crise da industrialização brasileira e com a crise energética mundial dos anos 7015 .

Em números gerais, existem, no Brasil, 8.763 assentamentos rurais, abrigando 924 mil famílias assentadas, numa área total de 85,8 milhões de hectares, segundo dados do INCRA em 2010 (Reforma Agrágria e Assentamentos. http:/ /www. portal.mda.gov.br/.../Reforma\%20Agraria\%20 e\%20Assentamentos\%20R.pdf, acessado em 20/ Nov/2012).

Dada a escassez de dados sobre as condições de saúde bucal dos assentados, o objetivo do presente estudo foi estimar a prevalência da autoavaliação negativa da saúde bucal e identificar possíveis fatores associados, em uma amostra de adultos em áreas do Assentamento Rural Governador Miguel Arraes, no Estado de Pernambuco, Brasil.

\section{Métodos}

O presente estudo foi desenvolvido nas áreas do Assentamento Rural Governador Miguel Arraes, que abrange os municípios de Água Preta, Catende, Jaqueira, Palmares e Xexéu, pertencentes a III GERES (Gerência Regional de Saúde) - Palmares, 
localizados na mesorregião da zona da mata do Estado de Pernambuco, com uma população geral de 24 mil habitantes.

Trata-se de um estudo transversal, constituído por uma população de referência de 4.235 indivíduos de 20 a 59 anos, residentes em 48 áreas de assentamento rural, realizado entre outubro de 2010 e janeiro de 2011. A amostra, do tipo probabilística, foi calculada com base nos seguintes parâmetros: nível de 95\% de confiança, erro-padrão de $5 \%$, poder de $80 \%$ de detectar uma diferença mínima de $20 \%$ entre grupos e prevalência de percepção negativa da saúde bucal de $50 \%$. A amostra final, acrescida de um fator de correção (efeito de agregação) de 1,2 e de $20 \%$ de perdas, correspondeu a 651 indivíduos.

O processo de amostragem foi por conglomerado, em duplo estágio. No primeiro estágio, as famílias foram sorteadas, previamente à coleta de dados, a partir de listas fornecidas de todos os beneficiários do assentamento disponibilizadas no sistema de informações de projetos de reforma agrária do INCRA - Regional Pernambuco. A princípio, foram sorteadas 12 famílias para cada uma das 48 áreas do Assentamento Rural Governador Miguel Arraes. No segundo estágio, foram sorteados os indivíduos que participaram do estudo, ou seja, em cada domicílio, foi selecionado um indivíduo com equiprobabilidade entre os moradores adultos (idade igual ou superior a 20 anos) para participar do estudo. Desta forma, as unidades amostrais primárias e secundárias foram, respectivamente, as famílias e os indivíduos.

Os critérios de inclusão foram: ter idade igual ou superior a 20 anos e ser morador das áreas do Assentamento Rural Governador Miguel Arraes. Foram excluídos da amostra os indivíduos que apresentavam algum impedimento físico e/ ou mental para responderem a entrevista e/ou participar do exame físico. Foi considerada perda amostral a pessoa que se recusou a participar da pesquisa, bem como os residentes ausentes em domicílios visitados, sem a obtenção de êxito por parte da pesquisadora, por, no mínimo, duas vezes, incluída ao menos uma visita em horário distinto ao anteriormente utilizado na primeira visita.

Os instrumentos utilizados na coleta de dados do presente estudo foram uma adaptação do questionário e formulário aplicados ao inquérito nacional de saúde bucal SBBrasil 2003 16. No questionário em específico, foram abordadas questões relacionadas à caracterização demográfica e socioeconômica, utilização dos serviços de saúde bucal, autopercepção da dor e da necessidade de tratamento odontológico, autoavaliação do atendimento odontológico e impacto da saúde bucal na qualidade de vida. Para o exame físico, foi utilizado um formulário específico para avaliação dos indivíduos em relação à doença cárie dentária, por meio do índice CPO-D; e para avaliação do edentulismo, pelo uso e necessidade de prótese; ambos os índices preconizados pela Organização Mundial da Saúde (OMS) 17 e utilizados no SBBrasil 200316.

O procedimento de calibração interexaminador foi realizado na Clínica da Atenção Básica da Faculdade de Odontologia da Universidade de Pernambuco pelo exame de duas sequências de 12 pacientes cada, apresentando as mais variadas condições clínicas, a fim de contemplar todos os critérios de diagnóstico. A concordância verificada pelo teste kappa $(\mathrm{k})$ indicou $\mathrm{k}=0,82$ (interexaminador). Para assegurar o controle de qualidade dos dados, cerca de $10 \%$ da amostra foi reexaminada - um a cada dez examinados era sorteado - conferindo uma concordância intraexaminador $\mathrm{k}=0,91$. Previamente à coleta de dados, realizou-se um estudo piloto envolvendo trinta indivíduos randomicamente selecionados, que não fizeram parte da pesquisa principal. Neste estudo, foram testados o questionário padronizado e o formulário específico para a realização do exame físico.

As entrevistas e os exames físicos foram realizados nos domicílios, por uma equipe de campo (uma cirurgiã-dentista e uma anotadora), sob luz natural por intermédio de inspeção tátil visual, com o auxílio de espelhos bucais planos e sondas periodontais tipo ball-point esterilizados. A equipe estava devidamente paramentada de acordo com as normas de controle de infecção (utilização de gorros, luvas e máscaras descartáveis).

A variável desfecho estudada, percepção da saúde bucal, foi obtida por meio da pergunta: "De modo geral, em comparação com as pessoas de sua idade, como o(a) Sr. (Sra.) considera seu estado de saúde bucal?". A percepção da saúde bucal foi caracterizada em cinco níveis: ótimo, bom, regular, ruim e péssimo. As categorias de resposta ótimo e bom foram agrupadas na categoria percepção positiva da saúde bucal e as categorias péssimo, ruim e regular foram consideradas como percepção negativa da condição de saúde bucal. Essa forma de operacionalização foi baseada nos estudos de Peek et al. 18 e Borrel et al. 19 .

É importante ressaltar que a categorização das variáveis independentes que se seguem foi elaborada ou por razões teóricas (literatura), ou por razões estatísticas (medidas de tendência central e de dispersão).

As variáveis independentes exploradas no presente estudo foram agrupadas de acordo com o modelo proposto por Gift et al. ${ }^{4}$ adaptado por 
Martins et al. 5, em: características demográficas; de predisposição/disponibilidade de recursos; comportamento relacionado à saúde bucal (uso de serviços odontológicos); condições objetivas de saúde bucal e condições subjetivas relacionadas à condição de saúde bucal. As primeiras compreenderam: faixa etária (20-29, 30-39, 40 49 e 50-59 anos); sexo (masculino/feminino) e cor da pele autorreferida (branca, negra, parda, amarela e indígena), a qual foi dicotomizada, em decorrência da homogeneidade da amostra, em branca e não-branca. A predisposição/disponibilidade de recursos foi avaliada pelas seguintes variáveis: escolaridade (analfabeto, fundamental incompleto, fundamental completo ou médio incompleto e médio completo ou superior); renda familiar (< 1 salário mínimo e $\geq 1$ salário mínimo); número de pessoas por domicílio (1-3, 4-6 e 7 ou mais pessoas) e orientações sobre como evitar problemas bucais (sim/não). O comportamento relacionado à saúde bucal foi mediado pelas variáveis: visita ao dentista ("Alguma vez na vida o Sr(a.) já foi ao consultório do dentista?”) ( $\operatorname{sim} /$ não); tempo decorrido após a última visita ao dentista ("Quando o $\operatorname{Sr}$ (a.) consultou o dentista pela última vez?") ( $<1,1-2, \geq 3$ anos) e motivo da visita ao dentista ("Qual o motivo da última consulta ao dentista?”) (consulta de rotina/revisão, dor, e tratamento odontológico).

As condições objetivas de saúde bucal foram mensuradas pelas variáveis: número de dentes permanentes cariados (0, 1-3, 4 ou mais dentes); número de dentes permanentes perdidos ( 0 , $1-19, \geq 20$ dentes); número de dentes permanentes obturados (0, 1-3, 3 ou mais dentes); uso de prótese (sim/não); necessidade de prótese (não necessita, superior, inferior, superior e inferior).

As condições subjetivas relacionadas à saúde bucal incluíram a percepção de dor ("Nos últimos seis meses o Sr(a.) sentiu dor de dente?”), para os casos positivos de dor, foi utilizada uma Escala Visual Analógica (EVA), consistindo em uma linha de $10 \mathrm{~cm}$, cujos limites estão marcados com os extremos da dor (nenhuma; pouca $(0-3 \mathrm{~cm})$; média/alta $(4-10 \mathrm{~cm})$; necessidade autorreferida de tratamento odontológico (sim/não); avaliação do atendimento na última visita ao dentista (ótimo/bom, regular, péssimo/ruim) e o impacto da condição de saúde bucal na qualidade de vida dos indivíduos por meio da forma simplificada do questionário Oral Health Impact Profile (OHIP-14) 20, variável esta dicotomizada em OHIP-14 = 0 (sem impacto) e OHIP-14 $\geq 1$ (com impacto).

Os dados da presente pesquisa foram duplamente digitados e foi realizada posterior checagem de inconsistências, utilizando-se o programa Epi Info 6.04 (Centers for Disease Control and
Prevention, Atlanta, Estados Unidos). As magnitudes das associações entre a variável desfecho e as variáveis independentes foram obtidas pelas razões de prevalências, com nível de significância de $5 \%$ e respectivos intervalos de $95 \%$ de confiança (IC95\%). As razões de prevalência bruta e ajustadas foram estimadas com o uso de regressão de Poisson, com ajuste robusto de variância, utilizando o teste de heterogeneidade de Wald. Para a análise estatística, utilizou-se o software Stata 10.0 (Stata Corp., College Station, Estados Unidos). As análises bivariadas da percepção de saúde bucal foram testadas usando o teste qui-quadrado de heterogeneidade e teste quiquadrado de tendência linear. Nesta análise, as variáveis com $\mathrm{p}<0,20$ foram selecionadas para o modelo multivariado. A análise ajustada foi realizada de acordo com um modelo hierárquico de determinantes em cinco níveis, nesta sequência: características demográficas; de predisposição; comportamento relacionado à saúde bucal (uso de serviços odontológicos); condições objetivas de saúde bucal e condições subjetivas relacionadas à condição de saúde bucal. A análise foi realizada de forma que o efeito das variáveis está controlado para aquelas do mesmo nível e as dos níveis superiores. Utilizou-se um nível de significância de $5 \%$ para o modelo multivariado.

O projeto foi aprovado pelo Comitê de Ética em Pesquisa da Universidade de Pernambuco, sob Parecer no 074/2010, com registro no Sistema Nacional de Informação sobre Ética em Pesquisa Envolvendo Seres Humanos (SISNEP - CAAE 0073.0.097.000-10).

\section{Resultados}

A proporção de resposta do presente estudo foi de $85,5 \%$. Os motivos para a não resposta foram: ausência nos domicílios nos dias pré-agendados $(n=35)$; recusa total em participar do estudo $(n=$ 25) e recusa parcial em participar do estudo ( $n=$ 34), ou seja, realizaram a entrevista, mas se recusaram a participar do exame físico.

A amostra total $(n=557)$ foi composta por $72,4 \%$ de mulheres $(n=403)$ e $27,6 \%$ de homens $(\mathrm{n}=154)$. A média de idade dos adultos foi de 32,8 anos. A maioria dos indivíduos (90,5\%) autodeclarou-se parda ou negra, sintetizada na categoria não branco. Não houve referência à raça/cor amarela ou indígena. Quanto ao nível de escolaridade da amostra, $64,3 \%$ dos indivíduos tinham menos de quatro anos de estudo, ou seja, haviam cursado o Ensino Fundamental de maneira incompleta, conforme Tabela 1.

Na Tabela 1, pode-se verificar que a prevalência geral estimada da percepção negativa da 
Tabela 1

Caracterização da amostra, prevalência de autoavaliação negativa da saúde bucal segundo características demográficas, de predisposição/disponibilidade de recursos, comportamento relacionado à saúde, condições objetivas e subjetivas, entre adultos, em áreas de assentamento rural. Pernambuco, Brasil, 2010/2011 ( N = 557).

\begin{tabular}{|c|c|c|c|}
\hline Variável & n (\%) & $\%$ & IC95\% \\
\hline \multicolumn{4}{|l|}{ Autoavaliação da saúde bucal } \\
\hline Positiva & $164(29,5)$ & & \\
\hline Negativa & $393(70,5)$ & & \\
\hline \multicolumn{4}{|l|}{ Características demográficas } \\
\hline \multicolumn{4}{|l|}{ Faixa etária (anos) } \\
\hline $20-29$ & $255(45,8)$ & 33,4 & $29,5-37,3$ \\
\hline $30-39$ & $156(28,0)$ & 20,3 & $16,9-23,6$ \\
\hline $40-49$ & $93(16,7)$ & 10,9 & $8,3-13,5$ \\
\hline $50-59$ & $53(9,5)$ & 5,9 & $3,9-7,9$ \\
\hline \multicolumn{4}{|l|}{ Sexo } \\
\hline Masculino & $154(27,6)$ & 20,1 & $16,7-23,4$ \\
\hline Feminino & $403(72,4)$ & 50,4 & $46,3-54,6$ \\
\hline \multicolumn{4}{|l|}{ Cor/Raça } \\
\hline Branco & $53(9,5)$ & 5,5 & $3,7-7,4$ \\
\hline Não branco & $504(90,5)$ & 65,0 & $61,0-68,9$ \\
\hline \multicolumn{4}{|l|}{ Predisposição/Disponibilidade de recursos } \\
\hline \multicolumn{4}{|l|}{ Escolaridade } \\
\hline Médio completo/Superior & $47(8,4)$ & 5,4 & $3,5-7,2$ \\
\hline Fundamental completo/Médio incompleto & $52(9,3)$ & 6,6 & $4,5-8,7$ \\
\hline Fundamental incompleto & $358(64,3)$ & 47,0 & $42,8-51,2$ \\
\hline Analfabeto & $100(18,0)$ & 11,5 & $8,8-14,1$ \\
\hline \multicolumn{4}{|l|}{ Renda familiar (salários mínimos) } \\
\hline$\geq 1$ & $540(97,0)$ & 68,6 & $64,7-72,4$ \\
\hline$<1$ & $17(3,0)$ & 1,9 & $0,8-3,1$ \\
\hline \multicolumn{4}{|l|}{ Número de pessoas por domicílio } \\
\hline $1-3$ & $174(31,3)$ & 21,7 & $18,3-25,1$ \\
\hline $4-6$ & $289(51,8)$ & 36,6 & $32,6-40,6$ \\
\hline 7 ou mais & $94(16,9)$ & 12,2 & $9,5-14,9$ \\
\hline \multicolumn{4}{|l|}{ Orientações sobre como evitar problemas bucais * } \\
\hline $\operatorname{Sim}$ & $242(50,1)$ & 35,0 & $30,7-39,2$ \\
\hline Não & $241(49,9)$ & 36,2 & $31,9-40,5$ \\
\hline \multicolumn{4}{|l|}{ Comportamento relacionado à saúde bucal } \\
\hline \multicolumn{4}{|l|}{ Visita ao dentista } \\
\hline Não & $15(2,7)$ & 1,2 & $0,3-2,1$ \\
\hline Sim & $542(97,3)$ & 69,3 & $65,4-73,1$ \\
\hline \multicolumn{4}{|c|}{ Tempo decorrido após a última visita ao dentista (anos) * } \\
\hline$<1$ & $248(45,8)$ & 31,7 & $27,8-35,6$ \\
\hline $1-2$ & $113(20,8)$ & 15,9 & $12,7-18,9$ \\
\hline$\geq 3$ & $181(33,4)$ & 23,6 & $20,0-27,1$ \\
\hline \multicolumn{4}{|l|}{ Motivo da visita ao dentista * } \\
\hline Consulta de rotina & $32(6,0)$ & 3,7 & $2,1-5,2$ \\
\hline Dor & $320(59,0)$ & 43,3 & $39,2-47,5$ \\
\hline Tratamento odontológico & $190(35,0)$ & 24,2 & $20,5-27,7$ \\
\hline
\end{tabular}

(continua) 
Tabela 1 (continuação)

\begin{tabular}{|c|c|c|c|}
\hline Variável & $n(\%)$ & $\%$ & IC95\% \\
\hline \multicolumn{4}{|c|}{ Condições objetivas relacionadas à saúde bucal } \\
\hline \multicolumn{4}{|c|}{ Número de dentes permanentes cariados } \\
\hline 0 & $143(25,7)$ & 14,2 & $11,3-17,0$ \\
\hline $1-3$ & $198(35,5)$ & 25,6 & $22,0-29,3$ \\
\hline 4 ou mais & $216(38,8)$ & 30,7 & $26,8-34,5$ \\
\hline \multicolumn{4}{|c|}{ Número de dentes permanentes perdidos } \\
\hline 0 & $48(8,6)$ & 5,4 & $3,5-7,2$ \\
\hline $1-3$ & $385(69,1)$ & 51,5 & $47,3-55,6$ \\
\hline 4 ou mais & $124(22,3)$ & 13,6 & $10,7-16,5$ \\
\hline \multicolumn{4}{|c|}{ Número de dentes permanentes obturados } \\
\hline 0 & $426(76,5)$ & 53,7 & $49,5-57,8$ \\
\hline $1-3$ & $90(16,1)$ & 11,6 & $9,0-14,3$ \\
\hline 4 ou mais & $41(7,4)$ & 5,2 & $3,3-7,0$ \\
\hline \multicolumn{4}{|l|}{ Uso de prótese } \\
\hline Sim & $246(44,1)$ & 29,6 & $25,8-33,4$ \\
\hline Não & $311(55,9)$ & 40,9 & $36,8-45,0$ \\
\hline \multicolumn{4}{|c|}{ Necessidade de prótese } \\
\hline Não necessita & $85(15,2)$ & 8,9 & $6,6-11,3$ \\
\hline Superior & $34(6,1)$ & 4,7 & $2,9-6,4$ \\
\hline Inferior & $143(25,7)$ & 19,4 & $16,1-22,6$ \\
\hline Superior/Inferior & $295(53,0)$ & 37,5 & $33,5-41,5$ \\
\hline \multicolumn{4}{|c|}{ Condições subjetivas relacionadas à saúde bucal } \\
\hline \multicolumn{4}{|l|}{ Percepção de dor * } \\
\hline Nenhuma & $332(59,8)$ & 38,3 & $34,3-42,4$ \\
\hline Pouca & $78(14,1)$ & 10,6 & $8,0-13,2$ \\
\hline Média/Alta & $145(26,1)$ & 21,6 & $18,2-25,0$ \\
\hline \multicolumn{4}{|c|}{ Necessidade autorreferida de tratamento * } \\
\hline Não & $72(13,4)$ & 4,6 & $2,8-6,4$ \\
\hline Sim & $467(86,6)$ & 66,9 & $63,0-70,9$ \\
\hline \multicolumn{4}{|c|}{ Avaliação do atendimento na última consulta odontológica * } \\
\hline Ótimo/Bom & $402(83,4)$ & 58,7 & $54,3-63,1$ \\
\hline Regular & $43(8,9)$ & 7,1 & $4,7-9,3$ \\
\hline Ruim/Péssimo & $37(7,7)$ & 5,4 & $3,3-7,4$ \\
\hline \multicolumn{4}{|l|}{ Impacto (OHIP-14) } \\
\hline Sem impacto & $94(16,8)$ & 5,9 & $3,9-7,8$ \\
\hline Com impacto & $463(83,2)$ & 64,6 & $60,6-68,6$ \\
\hline
\end{tabular}

IC95\%: intervalo de 95\% de confiança; OHIP-14: Oral Health Impact Profile.

* As diferenças nos valores absolutos das frequências correspondem às perdas de informação.

saúde bucal foi de $70,5 \%(\mathrm{n}=393)$. Ser do sexo feminino, estar na faixa etária mais jovem, ter se autodeclarado não branco, ser menos escolarizado foram características individuais que preponderaram nesta autopercepção negativa da condição de saúde bucal.

É possível observar, ainda na Tabela 2, que as variáveis que apresentaram associação estatisticamente significativa, por meio da análise bruta, foram: a cor/raça, visita ao dentista, número de dentes permanentes cariados, número de dentes permanentes perdidos, necessidade de prótese, percepção de dor, necessidade autorreferida de tratamento e impacto das condições de saúde bucal na qualidade de vida.

Por meio da análise multivariada, verificouse que as variáveis que permaneceram no modelo ajustado foram: a cor/raça, necessidade autorreferida de tratamento odontológico e impacto das condições de saúde bucal na qualidade de vida mediado pelo indicador OHIP-14. Desta forma, as pessoas que se autodeclararam não brancas autoavaliaram sua saúde bucal de maneira negativa $24 \%$ mais quando compara- 
Tabela 2

Prevalências e razões de prevalência (RP) bruta da autoavaliação negativa da saúde bucal segundo as características demográficas, predisposição/disponibilidade de recursos, comportamento relacionado à saúde bucal, condições objetivas e subjetivas relacionadas à saúde bucal, entre adultos, em áreas de assentamento rural. Pernambuco, Brasil, 2010/2011 $(\mathrm{N}=557)$.

\begin{tabular}{|c|c|c|c|}
\hline Variável & $\begin{array}{c}\text { Prevalência de } \\
\text { autoavaliação } \\
\text { negativa da } \\
\text { saúde bucal (\%) }\end{array}$ & RP (IC95\%) & Valor de $p$ \\
\hline \multicolumn{4}{|l|}{ Características demográficas } \\
\hline Faixa etária (anos) & & & 0,28 \\
\hline $20-29$ & $186(33,4)$ & $1,17(0,94-1,46)$ & \\
\hline $30-39$ & $113(20,3)$ & $1,16(0,92-1,47)$ & \\
\hline $40-49$ & $61(10,9)$ & $1,05(0,82-1,36)$ & \\
\hline $50-59$ & $33(5,9)$ & 1,00 & \\
\hline Sexo & & & 0,48 \\
\hline Masculino & $112(20,1)$ & $1,04(0,93-1,17)$ & \\
\hline Feminino & $281(50,4)$ & 1,00 & \\
\hline \multicolumn{4}{|l|}{ Cor/Raça } \\
\hline Branco & $31(5,5)$ & 1,00 & 0,04 \\
\hline Não branco & $362(65,0)$ & $1,23(0,97-1,55)$ & \\
\hline \multicolumn{4}{|l|}{ Predisposição/Disponibilidade de recursos } \\
\hline Escolaridade & & & 0,23 \\
\hline Médio completo/Superior & $30(5,4)$ & 1,00 & \\
\hline Fundamental completo/Médio incompleto & $37(6,6)$ & $1,11(0,85-1,47)$ & \\
\hline Fundamental incompleto & $262(47,0)$ & $1,15(0,92-1,43)$ & \\
\hline Analfabeto & $64(11,5)$ & $1,00(0,77-1,30)$ & \\
\hline Renda familiar (salários mínimos) & & & 0,59 \\
\hline$\geq 1$ & $382(68,6)$ & $1,09(0,77-1,56)$ & \\
\hline$<1$ & $11(1,9)$ & 1,00 & \\
\hline Número de pessoas por domicílio & & & 0,89 \\
\hline $1-3$ & $121(21,7)$ & 1,00 & \\
\hline $4-6$ & $204(36,6)$ & $1,02(0,90-1,15)$ & \\
\hline 7 ou mais & $68(12,2)$ & $1,04(0,89-1,22)$ & \\
\hline Orientações sobre como evitar problemas bucais & & & 0,50 \\
\hline $\operatorname{Sim}$ & $169(35,0)$ & 1,00 & \\
\hline Não & $175(36,2)$ & $1,04(0,93-1,16)$ & \\
\hline \multicolumn{4}{|l|}{ Comportamento relacionado à saúde bucal } \\
\hline Visita ao dentista & & & 0,04 \\
\hline Não & $7(1,2)$ & 1,00 & \\
\hline $\operatorname{Sim}$ & $386(69,3)$ & $1,53(0,89-2,63)$ & \\
\hline Tempo decorrido após a última visita ao dentista (anos) * & & & 0,41 \\
\hline$<1$ & $172(31,7)$ & 1,00 & \\
\hline $1-2$ & $86(15,9)$ & $1,10(0,96-1,25)$ & \\
\hline$\geq 3$ & $128(23,6)$ & $1,02(0,90-1,16)$ & \\
\hline Motivo da visita ao dentista * & & & 0,29 \\
\hline Consulta de rotina & $20(3,7)$ & 1,00 & \\
\hline Dor & $235(43,3)$ & $1,18(0,89-1,55)$ & \\
\hline Tratamento odontológico & $131(24,2)$ & $1,10(0,83-1,47)$ & \\
\hline
\end{tabular}

(continua) 
Tabela 2 (continuação)

\begin{tabular}{|c|c|c|c|}
\hline Variável & $\begin{array}{c}\text { Prevalência de } \\
\text { autoavaliação } \\
\text { negativa da } \\
\text { saúde bucal (\%) }\end{array}$ & RP (IC95\%) & Valor de $p$ \\
\hline \multicolumn{4}{|l|}{ Condições objetivas relacionadas à saúde bucal } \\
\hline Número de dentes permanentes cariados & & & $<0,001$ \\
\hline 0 & $79(14,2)$ & 1,00 & \\
\hline $1-3$ & $143(25,6)$ & $1,31(1,10-1,55)$ & \\
\hline 4 ou mais & $171(30,7)$ & $1,43(1,22-1,69)$ & \\
\hline Número de dentes permanentes perdidos & & & $<0,01$ \\
\hline 0 & $30(5,4)$ & 1,00 & \\
\hline $1-3$ & $287(51,5)$ & $1,02(0,79-1,32)$ & \\
\hline 4 ou mais & $76(13,6)$ & $1,22(1,05-1,42)$ & \\
\hline Número de dentes permanentes obturados & & & 0,92 \\
\hline 0 & $299(53,7)$ & 1,00 & \\
\hline $1-3$ & $65(11,6)$ & $1,03(0,89-1,19)$ & \\
\hline 4 ou mais & $29(5,2)$ & $1,01(0,82-1,24)$ & \\
\hline Uso de prótese & & & 0,10 \\
\hline Sim & $165(29,6)$ & 1,00 & \\
\hline Não & $228(40,9)$ & $1,09(0,98-1,22)$ & \\
\hline Necessidade de prótese & & & 0,04 \\
\hline Não necessita & $50(8,9)$ & 1,00 & \\
\hline Superior & $26(4,7)$ & $1,30(1,00-1,68)$ & \\
\hline Inferior & $108(19,4)$ & $1,28(1,05-1,57)$ & \\
\hline Superior/Inferior & $209(37,5)$ & $1,20(0,99-1,46)$ & \\
\hline \multicolumn{4}{|l|}{ Condições subjetivas relacionadas à saúde bucal } \\
\hline Percepção de dor * & & & $<0,001$ \\
\hline Nenhuma & $213(38,3)$ & 1,00 & \\
\hline Pouca & $59(10,6)$ & $1,18(1,02-1,37)$ & \\
\hline Média/Alta & $120(21,6)$ & $1,29(1,16-1,44)$ & \\
\hline Necessidade autorreferida de tratamento * & & & $<0,001$ \\
\hline Não & $25(4,6)$ & 1,00 & \\
\hline Sim & $361(66,9)$ & $2,23(1,62-3,07)$ & \\
\hline Avaliação do atendimento na última consulta & & & 0,48 \\
\hline \multicolumn{4}{|l|}{ odontológica * } \\
\hline Ótimo/Bom & $283(58,7)$ & 1,00 & \\
\hline Regular & $34(7,1)$ & $1,12(0,95-1,33)$ & \\
\hline Ruim/Péssimo & $26(5,4)$ & $1,00(0,80-1,24)$ & \\
\hline Impacto (OHIP-14) & & & $<0,001$ \\
\hline Sem impacto & $33(5,9)$ & 1,00 & \\
\hline Com impacto & $360(64,6)$ & $2,21(1,68-2,93)$ & \\
\hline
\end{tabular}

IC95\%: intervalo de 95\% de confiança; OHIP-14: Oral Health Impact Profile.

* As diferenças nos valores absolutos das frequências correspondem às perdas de informação.

das aos indivíduos que se declararam brancos; em relação à necessidade autorreferida de tratamento odontológico, esse percentual foi de $94 \%$ quando comparado àqueles que não perceberam a necessidade de tratamento; e, por fim, as pessoas que relataram alguma interferência da saúde bucal na qualidade de vida autoavaliaram sua saúde bucal de maneira negativa $82 \%$ mais em relação àquelas sem impacto, de acordo com a Tabela 3. 
Análise multivariada dos fatores associados à autoavaliação negativa da saúde bucal, entre adultos, em áreas de assentamento rural. Pernambuco, Brasil, 2010/2011 ( $N=557$ ).

\begin{tabular}{|c|c|c|c|c|c|c|}
\hline \multirow[t]{3}{*}{ Variável } & \multicolumn{6}{|c|}{ Autoavaliação negativa da saúde bucal } \\
\hline & \multicolumn{3}{|c|}{ Modelo bruto } & \multicolumn{3}{|c|}{ Modelo ajustado * } \\
\hline & RP & IC95\% & Valor de $p$ & RP & IC95\% & Valor de $p$ ** \\
\hline Cor/Raça (1) & & & 0,04 & & & 0,04 \\
\hline Branco & 1,00 & & & 1,00 & & \\
\hline Não branco & 1,23 & $0,97-1,55$ & & 1,24 & $1,00-1,53$ & \\
\hline Visita ao dentista (2) & & & 0,04 & & & 0,15 \\
\hline Não & 1,00 & & & 1,00 & & \\
\hline Sim & 1,53 & $0,89-2,63$ & & 1,50 & $0,86-2,53$ & \\
\hline Número de dentes permanentes cariados (3) & & & $<0,001$ & & & 0,31 \\
\hline 0 & 1,00 & & & 1,00 & & \\
\hline $1-3$ & 1,31 & $1,10-1,55$ & & 1,07 & $0,92-1,24$ & \\
\hline 4 ou mais & 1,43 & $1,22-1,69$ & & 1,12 & $0,97-1,30$ & \\
\hline Número de dentes permanentes perdidos (3) & & & $<0,01$ & & & 0,76 \\
\hline 0 & 1,00 & & & 1,00 & & \\
\hline $1-3$ & 1,02 & $0,79-1,32$ & & 1,14 & $0,79-1,65$ & \\
\hline 4 ou mais & 1,22 & $1,05-1,42$ & & 1,02 & $0,90-1,20$ & \\
\hline Uso de prótese (3) & & & 0,10 & & & 0,56 \\
\hline Sim & 1,00 & & & 1,00 & & \\
\hline Não & 1,09 & $0,98-1,22$ & & 1,03 & $0,92-1,16$ & \\
\hline Necessidade de prótese (3) & & & 0,04 & & & 0,28 \\
\hline Não necessita & 1,00 & & & 1,00 & & \\
\hline Superior & 1,30 & $1,00-1,68$ & & 1,25 & $0,89-1,76$ & \\
\hline Inferior & 1,28 & $1,05-1,57$ & & 1,20 & $0,90-1,61$ & \\
\hline Superior/Inferior & 1,20 & $0,99-1,46$ & & 1,11 & $0,83-1,48$ & \\
\hline Percepção de dor (4) & & & $<0,001$ & & & 0,58 \\
\hline Nenhuma & 1,00 & & & 1,00 & & \\
\hline Pouca & 1,18 & $1,02-1,37$ & & 0,99 & $0,86-1,15$ & \\
\hline Média/Alta & 1,29 & $1,16-1,44$ & & 1,05 & $0,95-1,16$ & \\
\hline Necessidade autorreferida de tratamento (4) & & & $<0,001$ & & & $<0,001$ \\
\hline Não & 1,00 & & & 1,00 & & \\
\hline Sim & 2,23 & $1,62-3,07$ & & 1,94 & $1,41-2,67$ & \\
\hline Impacto (OHIP-14) (4) & & & $<0,001$ & & & $<0,001$ \\
\hline Sem impacto & 1,00 & & & 1,00 & & \\
\hline Com impacto & 2,21 & $1,68-2,93$ & & 1,82 & $1,39-2,37$ & \\
\hline
\end{tabular}

IC95\%: intervalo de 95\% de confiança; OHIP-14: Oral Health Impact Profile; RP: razão de prevalência.

* Variáveis ajustadas entre o mesmo nível e pelos níveis anteriores: nível (1): características demográficas; nível (2): comportamentos relacionados à saúde bucal; nível (3): condições objetivas relacionadas à saúde bucal; nível (4): condições subjetivas relacionadas à saúde bucal;

** Teste de heterogeneidade de Wald.

\section{Discussão}

A relevância deste estudo deve-se ao fato de se tratar de pesquisa realizada em domicílios com uma amostra aleatória de adultos residentes em áreas de assentamento rural. Desta forma, ressalta-se a importância de se conhecer as con- dições de saúde bucal da população rural brasileira em razão da escassez de publicações acerca deste contingente populacional, que representa 15,65\% (aproximadamente, 30 milhões de pessoas) da população brasileira, segundo o censo do Instituto Brasileiro de Geografia e Estatística em 2010 (IBGE. Censo Demográfico de 2010. http:// 
www.censo2010.ibge.gov.br, acessado em 08/ Ago/2012).

A amostra do estudo foi caracterizada por predominância das mulheres, com idade média de 35,2 anos, de raça/cor autodeclarada não branca, com escolaridade baixa, vivendo em domicílios com um número médio de pessoas que variava entre quatro e seis pessoas, e renda familiar superior a um salário mínimo. Segundo o IBGE (http://www.censo2010.ibge.gov.br), a distribuição por gênero da população brasileira na zona rural em 2010 correspondeu a $47,3 \%$ de mulheres e $52,6 \%$ de homens, enquanto que, neste estudo, a proporção correspondeu a $72,4 \%$ de mulheres e $27,6 \%$ de homens. O controle desta variável não foi possível no momento da coleta de dados tendo em vista que consistiu de uma amostra domiciliar rural, e as entrevistas e exames físicos eram realizados durante o período matutino e vespertino, quando a maioria dos homens encontrava-se no trabalho.

No âmbito coletivo, os fatores demográficos e socioeconômicos podem contribuir para as variações de autoavaliação de saúde bucal entre os diferentes indivíduos da amostra estudada. No presente estudo, os indivíduos nas faixas etárias mais jovens julgaram mais negativamente seu estado de saúde bucal. No entanto, os resultados de estudos em relação à idade são inconsistentes neste tipo associação: alguns estudos 4,7 evidenciaram que a autoavaliação negativa da saúde bucal aumenta com a idade; em outro estudo 3 , entretanto, verificou-se que os mais velhos relatavam uma autoavaliação mais positiva da saúde bucal; e, em outro estudo 21, a idade não esteve associada à autoavaliação, convergindo com os achados da presente investigação. Corroborando outros estudos 4,7, não houve associação entre a autoavaliação negativa e o sexo, embora outras pesquisas 3,22 tenham evidenciado uma pior autoavaliação da saúde bucal entre as mulheres. Em relação à raça/cor, os resultados da presente investigação evidenciaram que os indivíduos que se autodeclararam brancos autoavaliaram sua saúde bucal de maneira mais positiva em relação aos não brancos, revelando uma associação estatisticamente significativa com autoavaliação negativa da saúde bucal, conforme evidenciam outros estudos 6,21. De fato, as iniquidades raciais relacionadas à saúde decorrem de violações continuadas, de longa data, dos seus direitos fundamentais, com reflexos diretos na percepção de saúde geral e bucal desses indivíduos.

As variáveis de predisposição e de disponibilidade de recursos pouco contribuíram para a identificação dos fatores associados à autoavaliação negativa da saúde bucal. Diversos estudos demonstram que, quanto maior a escolaridade do indivíduo 4,7,21 e o acesso a informações sobre como evitar problemas bucais 4,7 , mais positiva tende a ser a sua autoavaliação em saúde bucal. Na presente pesquisa, constatou-se uma melhor autoavaliação da saúde bucal entre os indivíduos de maior escolaridade, no entanto, esta variável não se mostrou associada com o desfecho. A literatura é controversa em relação à renda. A renda familiar reflete o poder de consumo do domicílio de forma mais acurada que a renda individual, por representar os possíveis ganhos de todos os membros do domicílio 23 , e diversos estudos $4,7,23$ evidenciam a relação entre maior renda familiar e autoavaliação positiva da saúde bucal. No presente estudo, essa associação não foi verificada devido à maior homogeneidade da população em relação à renda.

A utilização de serviços odontológicos traduz a autoavaliação da saúde, das necessidades, dos desejos e das demandas dos indivíduos, sendo mediada pela oferta, pelo acesso e pela qualidade dos serviços em uma dada sociedade 5 . No presente estudo, não houve associação entre a autoavaliação negativa da saúde bucal com as variáveis relacionadas à utilização de serviços odontológicos. No entanto, AfonsoSouza et al. 24 encontraram associação entre uma autoavaliação mais positiva da saúde bucal e o uso regular de serviços odontológicos. Na tentativa de explicar tal associação, os autores sugeriram duas hipóteses: que a autoavaliação positiva seria consequência de um bom tratamento, da maior oportunidade de receber tratamentos preventivos com reflexos positivos nas condições clínicas de saúde bucal; por outro lado, essa associação estaria relacionada à ocorrência de um bem-estar psicológico associado ao cuidado com a saúde.

Em relação às condições objetivas relacionadas à saúde bucal, a presente investigação observou que os indivíduos que tinham quatro ou mais dentes cariados, quatro ou mais dentes perdidos e que necessitavam de prótese superior estiveram mais fortemente associados, em termos de razões de prevalências, na análise bruta, com a autoavaliação negativa da saúde bucal, embora tal associação não tenha se mantido após o controle dessas pelas demais variáveis no modelo multivariado. De fato, Afonso-Souza et al. 24 afirmaram, com base nos achados de seu estudo, que a manutenção da dentição natural em condição satisfatória está associada com uma melhor autoavaliação da saúde bucal, e que a ausência de dentes naturais aumentava em quatro vezes a chance de classificar sua saúde bucal como ruim.

Dentre as variáveis relacionadas às condições subjetivas de saúde, a necessidade autorreferida 
de tratamento odontológico e o impacto das condições de saúde bucal na qualidade de vida estiveram fortemente associados à autopercepção negativa da saúde bucal, evidenciando, assim, que as medidas objetivas por si só são insuficientes para avaliar a saúde bucal. É importante salientar que a autopercepção da saúde bucal 8,20 e da saúde geral 2,6 muda ao longo da vida, do dia, da semana em função do seu estado físico e/ou psicológico e de condições contextuais. Sendo assim, mensurar a subjetividade do bem-estar e do adoecer, tentar quantificar o que é tão subjetivo é sempre limitado, pois envolve valores e sentimentos nem sempre expressos 8,11,13.

Neste artigo, verificou-se que foi alta a prevalência de autoavaliação negativa da saúde bucal entre os indivíduos das áreas do Assentamento Rural Miguel Arraes, e que os fatores que estiveram associados à autoavaliação negativa da saúde bucal foram a cor/raça; a necessidade autorreferida de tratamento odontológico e o impacto da saúde bucal na qualidade de vida. No entanto, é válido ressaltar que, para entender e discutir as interações entre autoavaliação da saúde bucal, a partir de modelos multidimensionais, como o proposto neste estudo, com características demográficas, com variáveis de predisposição/disponibilidade de recursos, com o comportamento relacionado à saúde bucal, bem como, as condições objetivas e subjetivas de saúde bucal entre a população rural brasileira, só será possível mediante o fortalecimento de bases conceituais e o incremento de investigações acerca do conhecimento das condições de saúde bucal deste contingente populacional, tendo em vista a lacuna existente na literatura em termos de publicações nesta área.

\section{Resumen}

El objetivo de este estudio fue estimar la prevalencia de autoevaluación negativa de salud bucal y factores asociados entre adultos en áreas de asentamiento rural. La muestra probabilística consistió en 557 adultos entre 20 a 59 años en áreas de asentamiento rural en el estado de Pernambuco, Brasil. La variable dependiente fue la autoevaluación de la condición de salud bucal y las independientes fueron: características demográficas, de predisposición/disponibilidad de recursos, comportamientos relacionados con la salud bucal, condiciones objetivas y subjetivas relacionadas con la salud bucal. Fueron estimadas las razones de prevalencia bruta y ajustada por medio de la regresión de Poisson. La prevalencia de autopercepción negativa de la salud bucal fue de un 70,5\%. La autoevaluación negativa de la salud bucal fue más prevalente en individuos más jóvenes, de baja escolaridad, entre las mujeres, $y$ entre los afrobrasileños y mestizos/mulatos. Los predictores de la autoevaluación negativa de la salud bucal fueron el color de la piel, la necesidad autorreferida de tratamiento odontológico y el impacto de las condiciones de salud bucal en la calidad de vida.

Autoevaluación; Población Rural; Salud Bucal;

Estudios Transversales; Adulto

\section{Colaboradores}

C. Moura, P. M. H. Santillo e R. S. C. Soares participaram da concepção e planejamento, introdução, interpretação dos dados, discussão e da revisão crítica do conteúdo. E. S. Gusmão e R. Cimões contribuíram na concepção e planejamento, introdução, interpretação dos dados, discussão e da revisão crítica do conteúdo, bem como da aprovação da versão final a ser publicada. 


\section{Referências}

1. Shields M, Shooshtari S. Determinants of selfperceived health. Health Rep 2001; 13:35-52.

2. Bezerra PCL, Opitz SP, Koifman RJ, Muniz PT. Percepção de saúde e fatores associados em adultos: inquérito populacional em Rio Branco, Acre, Brasil, 2007-2008. Cad Saúde Pública 2011; 27:2441-51.

3. Reisine ST, Bailit HL. Clinical oral health status and adult perceptions of oral health. Soc Sci Med 1980; 14:597-605.

4. Gift HC, Atchison KA, Drury TF. Perceptions of the natural dentition in the context of multiple variables. J Dent Res 1998; 77:1529-38.

5. Martins AMEBL, Barreto SM, Pordeus IA. Autoavaliação de saúde bucal em idosos: análise com base em modelo multidimensional. Cad Saúde Pública 2009; 25:421-35.

6. Barros MBA, Zanchetta LM, Moura EC, Malta DC. Auto-avaliação da saúde e fatores associados, Brasil, 2006. Rev Saúde Pública 2009; 43 Suppl 2:27-37.

7. Atchison KA, Gift HC. Perceived oral health in diverse sample. Adv Dent Res 1997; 11:272-80.

8. Martins AMEB, Barreto SM, Silveira MF, Santa-Rosa TTA, Pereira RD. Autopercepção da saúde bucal entre idosos brasileiros. Rev Saúde Pública 2010; 44:912-22.

9. Silva DD, Held RB, Torres SVS, Sousa MLR, Neri AL, Antunes JLF. Autopercepção da saúde bucal em idosos e fatores associados em Campinas, SP, 2008-2009. Rev Saúde Pública 2011; 45:1145-53.

10. Heaton LJ, Smith TA, Raybould TP. Factors influencing use of dental services in rural and urban communities: considerations for practitioners in underserved areas. J Dent Educ 2004; 68:1081-9.

11. Abreu MHNG, Pordeus IA, Modena CM. Representações sociais da saúde bucal entre mães no meio rural de Itaúna (MG), 2002. Ciênc Saúde Coletiva $2005 ; 10: 245-59$.

12. Cohen-Carneiro F, Rebelo MAB, Souza-Santos R, Ambrosano GMB, Salino AV, Pontes DG. Psycometric properties of the OHIP-14 and prevalence and severity of oral health impacts in rural riverine population in Amazonas State, Brazil. Cad Saúde Pública 2010; 26:1122-30.

13. Mendonça TC. Mutilação dentária: concepções de trabalhadores rurais sobre a responsabilidade pela perda dentária. Cad Saúde Pública 2001; 17:1545-7.

14. Guanziroli CE. Principais indicadores socioeconômicos dos assentamentos de reforma agrária. In: Romeiro A, Guanziroli C, Palmeira M, Leite S, organizadores. Reforma Agrária: produção, emprego e renda. O relatório da FAO em debate. Petrópolis: Editora Vozes; 1994. p. 13-68.
15. Coelho FMG, Castro TG, Campos FM, Campos MTFS, Priore SE, Franceschini SCC. Educação para promoção da saúde infantil: relato de experiência em um assentamento de reforma agrária, Vale do Rio Doce (MG). Ciênc Saúde Coletiva 2005; 10:739-47.

16. Departamento de Atenção Básica, Secretaria de Atenção à Saúde, Ministério da Saúde. Projeto SB Brasil 2003: condições de saúde bucal da população brasileira 2002-2003: resultados principais. Brasília: Ministério da Saúde; 2004. (Série C. Projetos, Programas e Relatórios).

17. World Health Organization. Oral health surveys: basic methods. $4^{\text {th }}$ Ed. Geneva: World Health Organization; 1997.

18. Peek CW, Gilbert GH, Duncan RP, Heft MW, Henretta JC. Patterns of change in self-reported oral health among dentate adults. Med Care 1999; 37:1237-48.

19. Borrel LN, Taylor GW, Borgnakke WS, Woolfolk MW, Nyquist LV. Perception of general and oral health in white and African American adults: assessing the effect of neighborhood socioeconomic conditions. Community Dent Oral Epidemiol 2004; 32:363-73.

20. Slade GD. Derivation and validation of a shortform oral health impact profile. Community Dent Oral Epidemiol 1997; 25:284-90.

21. Mathias RE, Atchison KA, Lubben JE, De-Jong F, Schweitzer SO. Factors affecting self-ratings of oral health. J Public Health Dent 1995; 55:197-204.

22. Mendonça HLC, Szwarcwald CL, Damacena GN. Autoavaliação de saúde bucal: resultados da Pesquisa Mundial de Saúde - Atenção Básica em quatro municípios do Estado do Rio de Janeiro, Brasil, 2005. Cad Saúde Pública 2012; 28:1927-38.

23. Mackenbach JP, Martikainen P, Leoman CW, Dalstra JA, Kunst AE, Lahelma E, et al. The shape of the relationship between income and self-assessed health: an international study. Int J Epidemiol 2010; 34:286-93.

24. Afonso-Souza G, Nadanovsky P, Chor D, Faerstein E, Weneck GL, Lopes CS. Association between routine visits for dental checkups and self-perceived oral health in na adult population in Rio de Janeiro: the Pró-Saúde Study. Community Dent Oral Epidemiol 2007; 35:393-4.

Recebido em 09/Ago/2012

Versão final reapresentada em 04/Dez/2012

Aprovado em 12/Dez/2013 